adjusting for age, sex, disease duration, total cumulative corticosteroid dose, and top 10 PCs as covariates. In further analysis of SLE patients with AVN compared with SLE patients without AVN treated by a high corticosteroid [cumulative dose $>20 \mathrm{~g}$, the AVN-risk loci were highly expressed in fibroblasts $(p=8.78 \times 105)$ and musculoskeletal system $(\mathrm{p}=1.13 \times 104)$ using DEPICT.

Conclusions These findings provide genetic evidence that pathway of fibroblasts are relevant to osteonecrosis. Individual genetic risk for the development of AVN should be assessed prior to treatment for SLE.

Funding Source(s): None

\section{INFLUENCE OF GENETIC RISK VARIANTS ON THE CLINICAL SUBPHENOTYPES OF SYSTEMIC LUPUS ERYTHEMATOSUS IN A KOREAN COHORT}

${ }^{1}$ Hyuk-Hee Kwon, ${ }^{2}$ Eunji Ha, ${ }^{1}$ So-Young Bang*, ${ }^{1}$ Hye-Soon Lee, ${ }^{2}$ Kwangwoo Kim, ${ }^{1}$ SangCheol Bae. 'Department of Rheumatology, Hanyang University Hospital for Rheumatic Diseases; ${ }^{2}$ Department of Biology, Kyung Hee University, Republic of Korea

\subsection{6/lupus-2019-Ism.136}

Background Systemic lupus erythematosus (SLE) is a multifactorial autoimmune disease with diverse heterogeneous phenotypes. Impact of complex genetic variants on the clinical manifestations of SLE was not fully understood. The aim of this study is to identify the cumulative effect of SLE-risk variants on clinical manifestations and autoantibody profiles in patients with SLE.

Methods A total of 781 Korean patients from the Hanyang BAE lupus cohort were genotyped by Immunochip and genome-wide association study (GWAS) using Illumina HumanOmni1-Quad array. Weighted genetic risk score (GRS) were calculated from 45 well-validated non-HLA SNPs and HLA SLE-risk loci. Individual GRS was tested for associations with the clinical subphenotypes based on 1997 ACR criteria of SLE and the development of autoantibody by using a linear regression or a logistic regression adjusting for the top 10 PCs as covariates, respectively.
Results We identified weighted GRS calculated from non-HLA and HLA SLE-risk loci with significant associations in various SLE subphenotypes defined by the ACR criteria (mean number 5.74) among 11 criteria. Individuals weighted GRS showed significantly positive correlation with the number of ACR criteria in a linear regression model (coefficient $=0.128$, $\mathrm{p}=9.00 \times 103)$. Consistently, a significant positive correlation with the number of ACR in both non-HLA GRS (coefficient $=0.110, p=0.027$ ) and HLA GRS (coefficient=0.06, $\mathrm{p}=0.021)$ was observed, respectively.

In a clinical subphenotype analysis, weighted GRS from non-HLA and HLA risk loci were significantly related to malar rash $(\mathrm{OR} 1.23, \mathrm{p}=2.68 \times 103)$, renal disorder $(\mathrm{OR} 1.15$, $\mathrm{p}=4.41 \times 102)$, and thrombocytopenia (OR 1.21, $\mathrm{p}=7.55 \times 103$ ) using a multivariable logistic regression. Weighted GRS were strongly associated with production of anti-DNA antibody (OR 1.38, p=2.18 $\times 103$ ).

Conclusions In conclusion, a high cumulative SLE-risk effect promoted diverse clinical manifestations and influenced the production of auto-antibodies including anti-DNA, that could provide clues to identifying distinctive mechanisms in SLE.

Funding Source(s): None

\section{MYOCARDITIS IN PEDIATRIC LUPUS: A CLINICAL CONUNDRUM}

${ }^{1}$ Nameirakpam Johnson*, ${ }^{2}$ Deepti Suri, ${ }^{3}$ Anju Gupta, ${ }^{3}$ Amit Rawat, ${ }^{2}$ Surjit Singh. ${ }^{1}$ Dept. of Pediatrics, Allergy- Immunology Unit, Postgraduate Institute of Medical Education and Research; ${ }^{2}$ Postgraduate Institute of Medical Education and Research, Chandigarh, India; ${ }^{3}$ PGIMER Chandigarh

\subsection{6/lupus-2019-Ism. 137}

Background Myocarditis in pediatric lupus is uncommon but carry a significant morbidity and mortality.

Methods We reviewed our cohort of children with lupus registered between January 1993 to November 2018. Of the 140 lupus patients that were diagnosed during this time, 4 had myocarditis.

Abstract 137 Table 1 Laboratory Investigations

\begin{tabular}{|c|c|c|c|c|}
\hline Investigation & Case 1 & Case 2 & Case 3 & Case 4 \\
\hline Haemoglobin $(\mathrm{g} / \mathrm{L})$ & $94 \mathrm{~g} / \mathrm{L}$ & $92 \mathrm{~g} / \mathrm{L}$ & $80 \mathrm{~g} / \mathrm{L}$ & $69 \mathrm{~g} / \mathrm{L}$ \\
\hline White cell counts & $6.7 \times 10^{9} / \mathrm{L}$ & $7.2 \times 10^{9} / \mathrm{L}$ & $6.5 \times 10^{9} / \mathrm{L}$ & $6.4 \times 10^{9} / \mathrm{L}$ \\
\hline Lymphocyte count & $1.15 \times 10^{9} / \mathrm{L}$ & $1.91 \times 10^{9} / \mathrm{L}$ & $1.65 \times 10^{9} / \mathrm{L}$ & $1.25 \times 10^{9} / \mathrm{L}$ \\
\hline Platelets & $130 \times 10^{9} / \mathrm{L}$ & $150 \times 10^{9} / \mathrm{L}$ & $220 \times 10^{9} / \mathrm{L}$ & $90 \times 10^{9} / \mathrm{L}$ \\
\hline Urine routine & No $R B C$, & No $R B C$, & No $\mathrm{RBC}$, & Plenty RBCs, 3+albumin \\
\hline Urine protein $\left(\mathrm{mg} / \mathrm{m}^{2} /\right.$ hour $)$ & No albumin & No albumin & No albumin & $82 \mathrm{mg} / \mathrm{m}^{2} / \mathrm{hour}$ \\
\hline C3 (Normal 50-150 mg/dL) & $27 \mathrm{mg} / \mathrm{dl}$ & $28 \mathrm{mg} / \mathrm{dL}$ & $31 \mathrm{mg} / \mathrm{dl}$ & $21 \mathrm{mg} / \mathrm{dl}$ \\
\hline C4 (Normal 20-50 mg/dL) & $4 \mathrm{mg} / \mathrm{dl}$ & $3 \mathrm{mg} / \mathrm{dL}$ & $10 \mathrm{mg} / \mathrm{dl}$ & $3 \mathrm{mg} / \mathrm{dl}$ \\
\hline ANA & 4+diffuse & 3+diffuse & $3+$ diffuse & 4+diffuse \\
\hline Anti dsDNA (N:<25 IU/mL) & 59 & 583 & 112 & 346 \\
\hline Antiphospholipid antibodies: & Positive & Negative & Negative & Negative \\
\hline a) Lupus anticoagulant & Negative & Negative & Negative & Negative \\
\hline $\begin{array}{l}\text { b) Anticardiolipin antibody (IgG and } \lg M \text { ) c) Anti B2 } \\
\text { Glycoprotein }-1 \text { antibody (IgG and } \lg M \text { ) }\end{array}$ & Negative & Negative & Negative & Negative \\
\hline 2Dimensional Echocardiography & $\begin{array}{l}\text { Mild pericardial effusion, } \\
\text { dilated left ventricle, ejection } \\
\text { fraction (EF) } 30 \%\end{array}$ & $\begin{array}{l}\text { Mild pericardial } \\
\text { effusion, EF } 25 \%\end{array}$ & $\begin{array}{l}\text { Mild pericardial effusion, } \\
\text { moderate mitral regurgitation, } \\
\text { mild tricuspid regurgitation, global } \\
\text { hypokinesia, EF } 56 \%\end{array}$ & $\begin{array}{l}\text { Moderate mitral regurgitation, } \\
\text { mild tricuspid regurgitation, global } \\
\text { hypokinesia, EF } 18 \%\end{array}$ \\
\hline
\end{tabular}


Results Patient 1 was an 11-year-old girl who presented with fever, rash and generalized body swelling for 3 months. Physical examinations revealed heart rate $148 / \mathrm{min}$, respiratory rate $30 / \mathrm{min}$, and blood pressure (BP) $100 / 80 \mathrm{mmHg}$. She had pallor, malar rash, hepatomegaly and gallop rhythm. Investigations are detailed in the table. She received intravenous immunoglobulin and initiated on pulse methylprednisolone and followed by pulse intravenous cyclophosphamide. She was discharged on oral prednisolone and subsequently tapered. Patient 2 was a 9-year-old girl presented with fever and rash for 3 months with lethargy for 1 day. She had pallor, malar rash, hepatomegaly. Physical examinations revealed heart rate $132 / \mathrm{min}$, respiratory rate $32 / \mathrm{min}$, feeble peripheral pulses and BP 100/80 mmHg. She received intravenous cyclophosphamide monthly for 6 months along with oral prednisolone in tapering dose. Patient 3 was a 12-year-old girl, who was known to have SLE for 2 months, presented with fever, malar rash, and photosensitivity. She had stopped taking steroids for 2 weeks. On examination, she had heart rate $122 / \mathrm{min}$, respiratory rate $28 / \mathrm{min}$ and $\mathrm{BP} 100 / 72 \mathrm{mmHg}$. She had pallor, alopecia, oral ulcers, malar rash, gallop rhythm and tender hepatomegaly. She was given pulse methylprednisolone and furosemide therapy followed by oral prednisolone in gradually tapering dose. Patient 4 was an 11 year-old-girl who was known to have SLE for 6 months, presented with pain in the small joints, low grade fever, and cough for 1 month. She had stopped steroids on her own. On examination, she was found to have anasarca, malar rash and oral ulcers. She had heart rate $128 / \mathrm{min}$, respiratory rate $32 / \mathrm{min}$, BP $108 /$
$74 \mathrm{mmHg}$, soft systolic murmur at the apex with normal heart sounds and hepatosplenomegaly. She was given pulse methylprednisolone along with cyclophosphamide in view of severity of symptoms. She developed multiorgan dysfunction and died after 8 days in hospital.

Conclusions Myocarditis in lupus is uncommon but carries a significant morbidities. The presence of global hypokinesia with a low ejection fraction on two dimensional echocardiography (2DE) is a strong pointer towards the diagnosis. Immunosuppressant drugs with supportive therapy remain the preferred treatment. Early diagnosis and prompt treatment may be very rewarding.

Funding Source(s): None

\section{RENAL OUTCOMES IN LUPUS NEPHRITIS RANDOMIZED CONTROLLED TRIALS: A SYSTEMATIC REVIEW AND A POOLED ANALYSIS ON 3922 PATIENTS}

Irene Cecchi*, Massimo Radin, Elisa Menegatti, Dario Roccatello, Savino Sciascia. University of Turin

\subsection{6/lupus-2019-Ism. 138}

Background Systemic lupus erythematosus (SLE) is a complex and potentially life-threatening autoimmune disease characterized by a wide range of manifestations. Renal involvement represents a major cause of morbidity and mortality and lupus nephritis (LN) remains a major therapeutic challenge. ${ }^{1}$ Therefore, selecting appropriate outcome measures for clinical trials

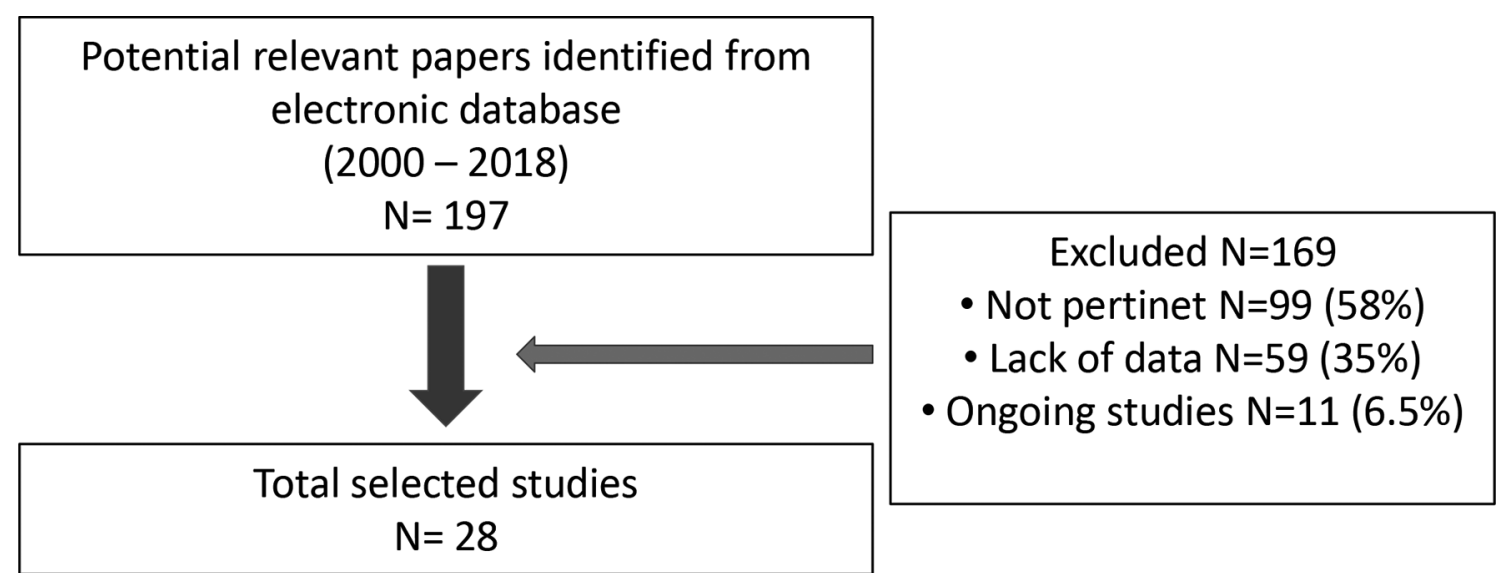
1. Chan et al (2000)
2. Houssiau et al (2002)
3. Contreras et al (2004)
4. Ginzler et al (2005)
5. Li et al (2009)
6. Appel et al (2009)
7. Zavada et al (2010)
8. Dooley et al (2011)
9. Chen et al (2001)
10. Zeher et al (2011)
11. Yap et al (2012)
12. Rovin et al (2012)
13. Houssiau et al (2012)
14. Mysler et al (2012)

15. Jayne et al (2013)

16. Furie et al (2014)

17. Mok et al (2014)

18. ACCESS trial group (2014)

19. Anutrakulchai et al (2015

20. Liu et al (2015)

21. Kaballo et al (2016)

22. Rovin et al (2016)

23. Rathi et al (2016)

24. Rovin et al (2018)

25. Furie et al (2018)

26. Mehra et al (2018)

27. Kamanamool et al (2018)

28. Sedhain et al (2018)

\section{Abstract 138 Figure 1}

